\title{
Inertial Navigation System for Mobile Land Vehicles
}

\author{
Jorge Lobo, Paulo Lucas, Jorge Dias, A. Traça de Almeida \\ ISR - Instituto de Sistemas e Robótica \\ Departamento de Engenharia Electrotécnica \\ Universidade de Coimbra \\ Largo Marquês de Pombal \\ 3000 COIMBRA, PORTUGAL \\ E-mail : jorge@mercurio.uc.pt \\ fax : +351-39-35672
}

\begin{abstract}
This paper describes a prototype of an inertial navigation system for use in mobile land vehicles, such as cars or mobile robots. The complete system is composed by sensors, their mechanical mount and cabling, these connect to a PC card with local processing and memory, based on a Intel 80C196KC microcontroller.
\end{abstract}

The sensors used were a piezoelectric vibrating gyroscope, two silicon accelerometers, two clinometers and also a magnetic fluxgate compass acting as an independent source for drift compensation. The working principle of the various sensors are presented.

The experimental setup used for testing is based on a robot arm controlled in real time to move the sensors along a known trajectory (velocity control).

\section{Introduction}

From inertial measurements we can determine an estimate for linear accelerations and angular velocities. By integrating these quantities we determine the velocity vector and the body attitude. Position can be calculated by integration of the velocity vector. Inertial navigation is thus based on the dead-reckoning principle.

Inertial navigation systems have been widely used in aerospace applications but are only now being seriously exploited in robotics applications where they have considerable potential. Recent development in solid-state accelerometers and gyrometers has lead to some new low-price sensors. These sensors, though not suitable for high performance inertial systems, can provide a reasonable system suitable for some applications. Unlike the typically high-cost systems developed for aerospace, these new systems are much more price-competetive and thus suitable for a wider range of applications. The needs of the automotive industry has made these systems increasingly commercially available. They lack the high accuracy of their aerospace counterparts, but by appropriate modelling of the system they can provide useful positioning information for many applications.

A theoretical system using three accelerometers and three gyroscopes was presented in [1]. Methods of extracting the motion and orientation of the system from inertial information are derived theoretically but not directly implemented in a real system. In [2] a vibrating beam gyroscope was used for short range orientation estimation for outdoor vehicles, enabling wider spacing of navigation beacons. In [3] an optical fiber gyroscope was used for position estimation, by combining the gyro data with the wheel encoders data. In [4] a calibration method is proposed for gyrometers, based on the use of a redundant one.

In this work we developed a prototype of an inertial navigation system for use in mobile land vehicles, such as cars or mobile robots. We hope to take advantage of the vehicles limited movements to build a simplified inertial system. Unlike some systems that rely on outdoor environments to obtain additional information, like GPS NavSat [5], or on a knowledge of the vehicle's moving space [6], we intend to build a system that is capable of working either indoors or outdoors in an unstructured environment. We assume however that $\vec{g}$ is a constant uniform 3$\mathrm{D}$ vector field.

The following sections describe the proposed system, the sensors used and their working principle, how the system was put together and the experimental setup used to test it.

\section{Proposed Inertial Navigation System}

The complete system is composed of the sensors, their mechanical mount and cabling, the data acquisition hardware and the controlling software.

\subsection{The Hardware}

The sensors used were a piezoelectric vibrating gyroscope, two silicon accelerometers, two clinometers and also a magnetic fluxgate compass acting as an independent source for drift compensation.

\subsubsection{Gyrometer}

In order to keep track of rotation on the z-axis a Gyrostar ENV011D, built by Murata, was used. The device is a sensor for angular velocity. Therefore the orientation must be calculated by integration of the angular velocity over time. The device's output 
is a voltage proportional to the angular velocity and relative to a reference.

The principle of the sensor is outlined in Fig. 1. Inside the device there is an equilateral triangle prism made from elastic invariable metal, which is fixed at two points. Three piezoelectric ceramic elements are attached to the faces of prism, one on each side. The prism is forced to vibrate at about $7 \mathrm{kHz}$ by one of the piezoelectric elements, whilst the other two are used for detection. When there is no rotation they detect equally large signals. When the prism is turned it gets twisted, so the detectors receive different signals. The difference between the signals is processed by the internal analog circuits to provide an output voltage proportional to the angular velocity [7].

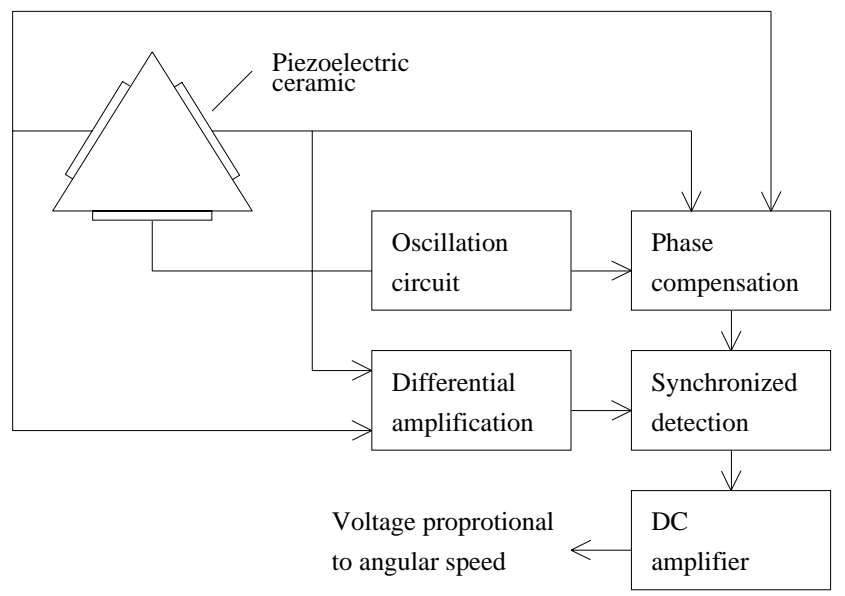

Fig.1. Block diagram of the piezoelectric vibrating gyroscope.

The Gyrostar ENV-011D has a high precision compared to other vibration gyroscopes. It is linear to within $\pm 0.5 \%$ of maximum angular velocity. Has an angular velocity range of \pm 90 $\mathrm{deg} / \mathrm{sec}$ and no hysteresis. The output DC voltage is proportional to angular rate by a $22.2 \pm 0.65 \mathrm{mV} / \mathrm{deg} / \mathrm{sec}$ scale factor and ranges from $0.5 \mathrm{~V}$ to $4.5 \mathrm{~V}$. Simpler models of the gyroscope built by Murata are known to have self-heating problems that cause offset drift [4]. Tests carried out with the ENV-011D showed it wasn't significantly affected by this problem. The drift is only noticeable when integrated over long periods of time. To overcome this problem, external references have to be used, such as the fluxgate compass included in our system and described in following section.

\subsubsection{Accelerometers}

Two piezoresistive accelerometers were used to measure accelerations along the $\mathrm{x}$ and $\mathrm{y}$ axis. The device used, 3145-002 model from ICSensors, is a double cantilever silicon accelerometer. The full scale output is \pm 2 volts about a 2.5 volt offset. The sensors used had a range of $\pm 2 \mathrm{~g}$.

Silicon sensor development has enabled the batch-fabrication of this integrated accelerometer. It incorporates amplification, signal conditioning, temperature compensation and internal voltage regulation, all in a small package. Piezoresistors are diffused onto the flexures of the bridge supporting the sensing mass. The resultant electrical structure is a Wheatstone bridge where two resistors increase with downward acceleration and two decrease with the same force. This provides a true DC response, unlike the piezoelectric accelerometers that only have AC response. The full bridge design substantially reduces off-axis sensitivity. Since sideways accelerations cause symmetric disturbances on each side of the double cantilever structure, their effect is cancelled out, provided the piezoresistors are well matched. A typical frequency response of the sensor is shown in Fig. 2.

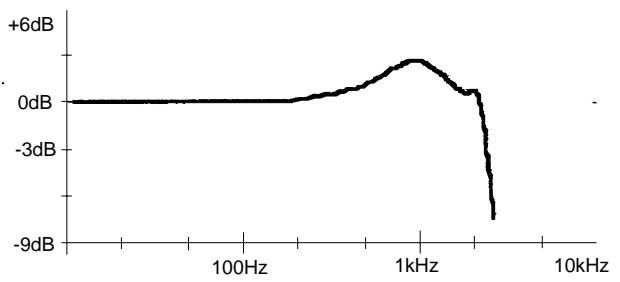

Fig.2. Accelerometer frequency response (from ICSensors calibration data).

As can be seen from the frequency response, the sensors used were slightly under-damped. When designing the acquisition filters this was taken into account, so as to avoid output saturation and distortion.

\subsubsection{Clinometers}

To measure tilt about the $\mathrm{x}$ and $\mathrm{y}$ axis two AccuStar electronic clinometers, built by Lucas Sensing Systems, were used. The device has a linear range of $\pm 45 \mathrm{deg}$. Cross-axis inclinations up to $45 \mathrm{deg}$ introduce an error of less than $1 \%$. The concept of the sensor is based on a dielectric fluid with an air bubble inside a capacitive sensor. When you tilt the sensor, the bubble, moving under the force of gravity, changes the capacitance of the sensor elements. The resulting differential generates an output signal which reflects the relative tilt in the sensing axis as shown in Fig. 3.

The clinometers have a delayed response with a time constant of 0.3 seconds. This is not a problem if taken into account in the calculations. Care should also be taken when accelerations other than gravity are present, since the tilt will be measured relative to the resultant vector. In our system we

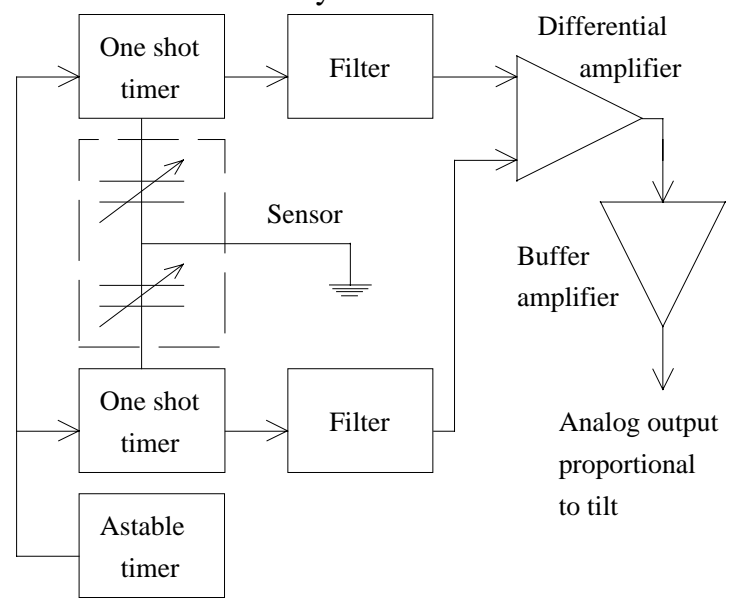

Fig.3. Block diagram of the capacitive clinometer. 
assume that, from time to time, we know that the vehicle is at rest, and can therefore keep track of the vertical.

\subsubsection{The Fluxgate Compass}

The compass used is a $\mathrm{C} 100$ model from KVH Industries. The fluxgate sensor element is a saturable ring core, free floating in an inert fluid within a cylindrical lexan housing. The purpose of the floating ring core is to keep the sensing element horizontal with respect to the earth. Hard mounted cores experience significant errors when not perfectly horizontal (often more than three degrees of error for each degree of tilt). The lexan housing is surrounded by windings which electrically drive the coil into saturation [8].

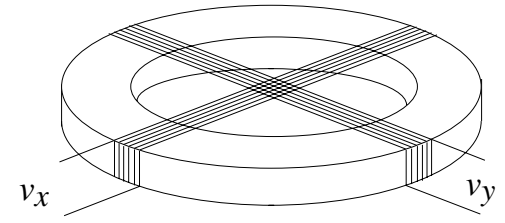

Fig.4. Flux-gate sensor element.

Pulses, whose amplitude is proportional to the sensed horizontal component of the earth's magnetic filed, are detected by two secondary windings that are at right angles, as can be seen in Fig. 4. These provide data on the $\mathrm{x}$ and $\mathrm{y}$ horizontal components of the earth's magnetic field. These signals are converted to a DC level, digitized and then sent to a microprocessor that calculates the azimuth angle as

$$
\phi=\tan ^{-1}\left(\frac{\mathrm{v}_{\mathrm{x}}}{\mathrm{v}_{\mathrm{y}}}\right)
$$

See [8] for more details.

The C100 compass can compensate only for moderate magnetic interferences. It is convenient to keep a distance of at least 12 inches [9] from iron, steel, magnets, motors, and other magnetic material. As this was incompatible with our application, especially the Puma560 used in experimental setup, a magnetic house shield was built. The material, the shape and the dimensions of the shield had to be studied. As the compass was cylindrical, that was the chosen geometry for the shield. A large sphere would be theoretically ideal, but the cylinder proved to be a good solution.

We wanted a material that had a good shield effectiveness for all magnetic interferences, but permeable to the earth magnetic field. The fact that the lower frequencies are harder to shield means that, in the end, the earth field gets through the shield. Several shielding effectiveness tables were consulted [10] [11]. These analysed the performance of various materials with different frequencies and source locations. Steel was found to be the more appropriate material since it has a good behaviour over a wide range of frequencies. From the different steels we selected SAE 1045 (AISI standard), or CK45 (DIN standard), for its excellent properties $\left(\mu_{\mathrm{r}}=1000\right.$ and $\left.\sigma_{\mathrm{r}}=0.1\right)$, for its availability and low cost.

The dimensions of the shielding material had to be small enough so as not to make the piece bulky and heavy, but big enough to provide reasonable shielding. The effectiveness of the shield is determined by tree factors, the absorption loss A, the reflection loss $\mathrm{R}$ and the thin shield correction parameter $\mathrm{B}$ (for multiple reflection of magnetic fields). Being the thickness of the shielding material and $f$ the magnetic field frequency we have $S$ $=\mathrm{A}+\mathrm{R}+\mathrm{B}$, i.e.

$$
\mathrm{S}=\left[3,34 \times 25,4 t \sqrt{f \mu_{r} \sigma_{r}}\right]+\left[14.6+10 \log \left(\frac{\mathrm{f}^{2} \sigma_{r}}{\mu_{\mathrm{r}}}\right)\right]+\mathrm{B}[\mathrm{dB}](
$$

For low frequencies, where the shield effectiveness is lower, the correction parameter B and the reflection loss of the selected steel are negligible, making the shield effectiveness determined only by the absorption loss. This is directly proportional to the thickness of the selected material, and consequently to its skin depth $\delta$, given by

$$
\delta=\frac{66,04}{\sqrt{f \mu_{r} \sigma_{r}}}[\mathrm{~mm}]
$$

For $\mathrm{t}=5 \delta$, the magnetic field reduces to $\mathrm{e}^{-t / \delta}|\overrightarrow{\mathrm{B}}|=0,67 \%|\overrightarrow{\mathrm{B}}|$. So for a $6 \mathrm{~mm}$ thick steel and an outside magnetic field of say $30 \mathrm{~Hz}$, the magnetic interference inside the shield is 149 times lower. The cylinder apertures drastically reduce the shield effectiveness. To avoid this the cylinder tops have an L cross-section at the point of contact, and the passage aperture for the compass wires follows the same criteria.

The built shield was a $80 \mathrm{~mm}$ high hollow cylinder with $6 \mathrm{~mm}$ walls and $40 \mathrm{~mm}$ radius. The compass was placed inside, in a way that the geometric centres of the two components matched.

The shield performed well in the carried out tests. The pointed north by the fluxgate compass changes, as we expected, because the shield is made of a ferromagnetic material that causes a constant interference in the compass, but the azimuths in respect to the new north are well computed. The tests also showed that the magnetic interference of the surrounding environment had reduced much, even for very small distances. So, we could have more confidence in the values obtained by the fluxgate compass.

The C100 compass has its own electronics controller board. Several output modes are available. A serial port is also present to perform compass configuration. The RS-232 port can also provide heading information.

For monitoring angular motion along the $\mathrm{z}$ axis the system already has a gyroscope. But since the gyro readings are prone to drift, the compass is called upon periodically to correct the drift. The analog and digital direct outputs provided by the compass are faster, but for our application, the serial port did just fine.

\subsubsection{Getting all sensors together}

The sensors had to be assembled so as to be properly aligned relative to each other and easily attached to the vehicle, or in the case of the test apparatus, the Puma560 robot arm. They were therefore mounted inside a cube with a suitable geometry for extracting the relevant data as can be seen in Fig. 5. It is made of $8 \mathrm{~mm}$ thick acrylic, having $120 \mathrm{~mm}$ inner side. The acrylic material was light enough to be attached to the end of the Puma560 robot arm, and rigid enough for holding the sensors at appropriate locations. 


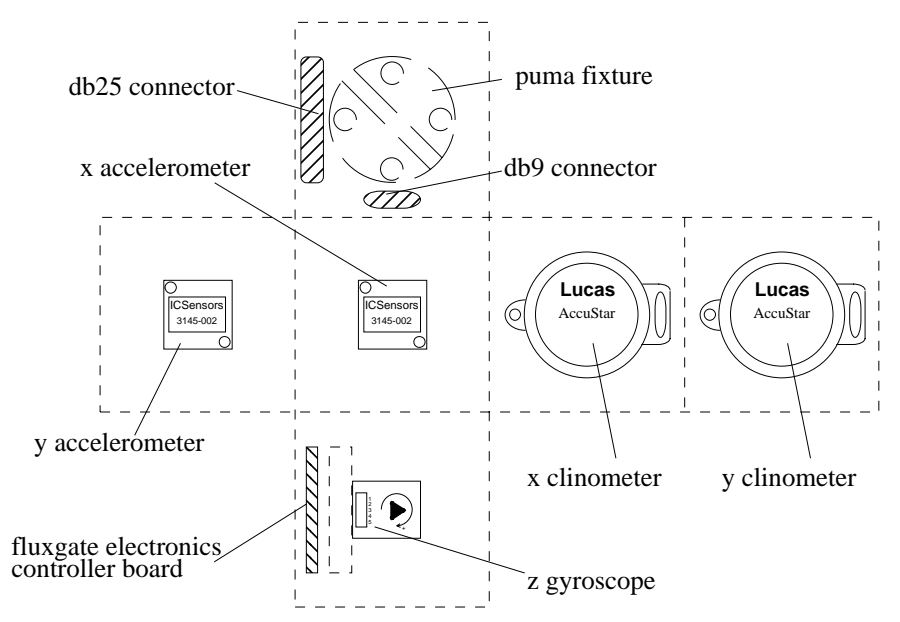

Fig.5. Flattened layout of the cube.

Since the sensors sensing point couldn't all coincide, they had to be placed at convenient locations. The gyroscope, provided it is correctly aligned with the z-body-axis, will always measure the correct angular speed. But the accelerometers, being away from the center of the cube, will be subject to centrifugal acceleration. This can be compensated for, provided the sensor is correctly aligned and the distance to the center is known.

The cube provided a modular and compact way of protecting the sensors, as well as housing all the connecting cables, while at the same time placing the sensors along the desired axis. The fluxgate compass was placed inside it's magnetic shielding cylinder and attached to the bottom of the cube, as can be seen in Fig. 8. The compass electronics board was placed inside the cube. The cube is connected by a multicore cable to the controller pcboard and by a RS-232 cable to the personal computer serial port.

\subsubsection{The processing hardware}

To handle the data acquisition, and also enable some processing, a micro-controller based card was built. The card is designed to work in a personal computer and has an EISA bus interface. The 16-bit 80C196KC micro-controller was chosen, since it provided the necessary control functions as well as reasonably fast data processing to implement the algorithms. The board provides power to the sensors and acquires the relevant data, with appropriate filtering and sampling. Figure 6 shows its architecture.

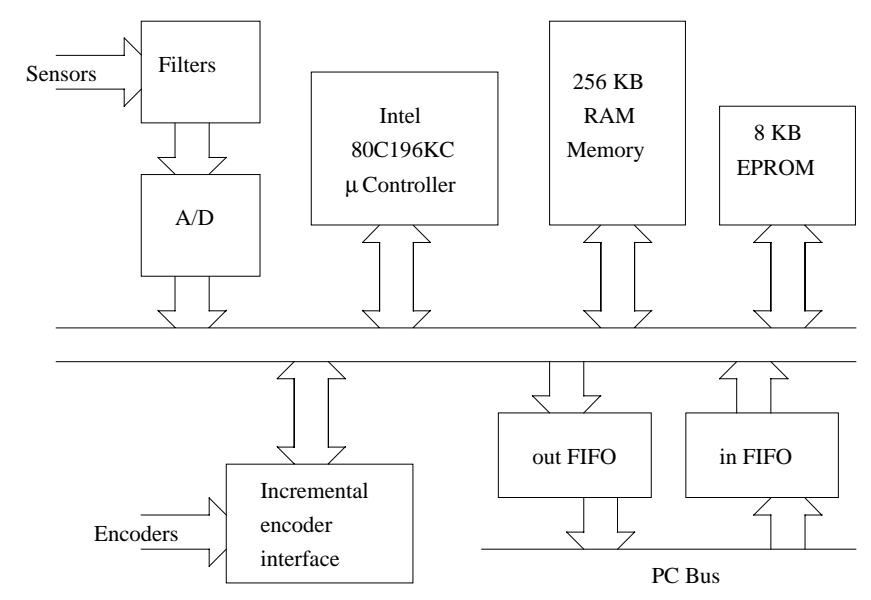

Fig.6. Architecture of the controller pcboard.

The sensors output are already in a convenient format, needing only anti-aliasing analog filters. An external A/D converter was used, enabling faster and higher precision sampling than the 80C196KC internal A/D, which was left for measuring less demanding signals.

The micro-controllers address space is only $64 \mathrm{~KB}$, in order to expand it, a windowing scheme was used. The EPROM stores the onboard software. To communicate with the PC two FIFOs were used. This allowed the onboard processing to continue without having to wait for the personal computer. In development stages the software can be downloaded from the personal computer to the onboard memory, using the EPROM to store only a simple monitoring program.

Although not part of the current system, an encoder interface was included in the design. This was done to extend the range of applications were the board could be used.

The fluxgate compass has its own electronics board with a RS-232 port that directly connects to the PC, as shown in Fig. 7.

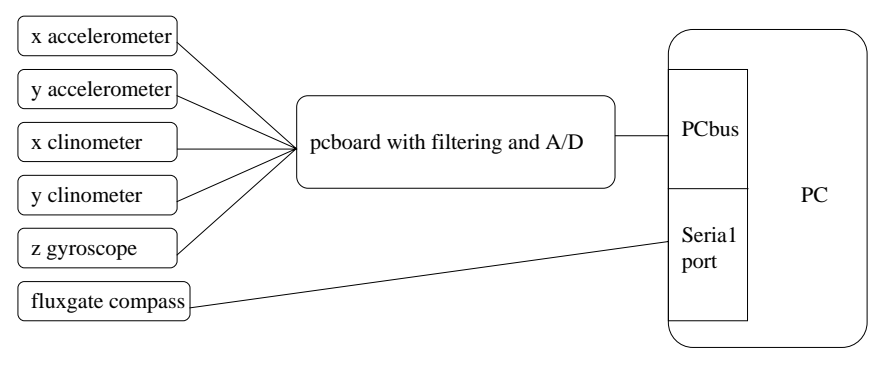

Fig.7. Layout of the system.

\subsection{Positioning Algorithms}

The system is still under development, and the first algorithm tried out is a simple solution for $2 \mathrm{D}$ navigation. A preliminary calibration is done by placing the cube at a levelled plane. The sensors' offsets are then measured, providing a zero-point reference for future readings.

Initially the cube is at rest and the clinometers give the direction of $\vec{g}$ relative to the body. With $\alpha_{\mathrm{x}}$ and $\alpha_{\mathrm{y}}$ being the sensed angles by the $\mathrm{x}$ and $\mathrm{y}$-axis clinometers, we have 


$$
\frac{\vec{g}}{\|\vec{g}\|}=\left[\begin{array}{c}
\sin \alpha_{y} \\
-\sin \alpha_{x} \\
-\sqrt{1-\sin ^{2} \alpha_{x}-\sin ^{2} \alpha_{y}}
\end{array}\right]
$$

given in a body frame of reference.

Provided that all future movements, until the next system reset, are done along a 2D plane, we can estimate the body's position with the data given by the accelerometers and the gyroscope.

The accelerometer will pick up some centrifugal acceleration due to the rotation $\omega$ about the body z-axis. This can be compensated for, since $\omega$ and the radius $r$ are known. This combination is given by

$$
\mathrm{a}_{\text {body }}=\mathrm{a}_{\text {sensed }}+\mathrm{r} \omega^{2}
$$

for both $\mathrm{x}$ and $\mathrm{y}$-axis accelerometers.

To keep track of the rotation around the $\mathrm{z}$-axis, the angular velocity $\omega$ sensed by the gyroscope must be integrated. Defining $\theta_{z}$ as the current z-axis angle relative to the original orientation we have

$$
\theta_{z}=\int_{0}^{T} \omega_{z} d t
$$

Our current system only deals with rotations about the body $\mathrm{Z}$ axis. The initial direction of $\dot{g}$ is known from (4). To keep track of it, we must update it to the rotated body frame of reference. Assuming a rotation angle $\theta_{z}$,

$$
\frac{\vec{g}}{\|\vec{g}\|}=\left[\begin{array}{ccc}
\cos \theta_{z} & -\sin \theta_{z} & 0 \\
\sin \theta_{z} & \cos \theta_{z} & 0 \\
0 & 0 & 1
\end{array}\right]\left[\begin{array}{c}
\sin \alpha_{y} \\
-\sin \alpha_{x} \\
-\sqrt{1-\sin ^{2} \alpha_{x}-\sin ^{2} \alpha_{y}}
\end{array}\right]
$$

The acceleration experienced by the body will be the sum of gravity with the true accelerations, i.e.

$$
\overrightarrow{\mathrm{a}}_{\text {true }}=\overrightarrow{\mathrm{a}}_{\text {sensed }}-\vec{g}
$$

As long as no $\mathrm{x}$ or $\mathrm{y}$-axis rotations occur (limited to a 2D plane), we can separate the two, since the direction of $\vec{g}$ is known from (7) and $\|\vec{g}\|$ is constant $\left(g=\|\vec{g}\| \cong 9.81 \mathrm{~m} / \mathrm{s}^{2}\right)$. The true body accelerations are then given by

$$
\begin{aligned}
& \mathrm{a}_{\mathrm{x}_{\text {true }}}=\mathrm{a}_{\mathrm{x}_{\text {body }}}-\mathrm{g}\left(\sin \alpha_{y} \cos \theta_{z}+\sin \alpha_{x} \sin \theta_{z}\right) \\
& \mathrm{a}_{\mathrm{y}_{\text {true }}}=\mathrm{a}_{\mathrm{y}_{\text {body }}}-\mathrm{g}\left(\sin \alpha_{y} \sin \theta_{z}-\sin \alpha_{x} \cos \theta_{z}\right) \\
& \mathrm{a}_{\mathrm{z}_{\text {true }}}=0 \quad \text { (assumed) }
\end{aligned}
$$

To convert to the navigation frame, defined by the body's initial attitude and position (last reset), we need to rotate the true acceleration vector by $-\theta_{z}$.

$$
\vec{a}_{\text {nav }}=\left[\begin{array}{ccc}
\cos \left(-\theta_{z}\right) & -\sin \left(-\theta_{z}\right) & 0 \\
\sin \left(-\theta_{z}\right) & \cos \left(-\theta_{z}\right) & 0 \\
0 & 0 & 1
\end{array}\right]\left[\begin{array}{c}
\mathrm{a}_{\mathrm{x}_{\text {true }}} \\
\mathrm{a}_{\mathrm{y}_{\text {true }}} \\
0
\end{array}\right]
$$

To obtain body position we only need to integrate (10). Assuming initial rest conditions,

$$
\vec{x}_{\text {nav }}=\iint_{0}^{T} \vec{a}_{\text {nav }} d t
$$

with $\vec{a}_{\text {nav }}=\left[\begin{array}{lll}0 & 0 & 0\end{array}\right]$ and $\vec{v}_{\text {nav }}=\left[\begin{array}{lll}0 & 0 & 0\end{array}\right]$.

We will therefore have the final position and attitude of the body given by

$$
\vec{x}_{\text {nav }}=(x, y) \text { and } \theta_{z}
$$

relative to initial position.

For movements along planes close to the level plane, the fluxgate compass can be used to compensate the accumulated drift in $\theta_{z}$. When the difference between the two estimates exceeds a preset value, the compass reading is taken as the correct one. But if the compass reading shows an abrupt change, we keep the value given by the gyroscope integration, since the fluxgate can be affected by bumpy accelerations. An absolute reset on the accumulated drift error is therefore provided.

\subsection{Experimental Setup}

The cube was attached to the end of a Puma560 robot arm (see. Fig. 8), enabling the movement of the sensors along a known trajectory (velocity control).

A software package was made to provide a set of tools for development and testing of the inertial navigation system. The software (INERworkbench) processes data off-line, that is, the trajectory control and data acquisition is done in real time but the algorithms are tested off-line, using the known trajectory to evaluate their accuracy. The INERworkbench allows the generation of composed trajectories, specifying the parameters for each path section, and their triggering and control in real time.

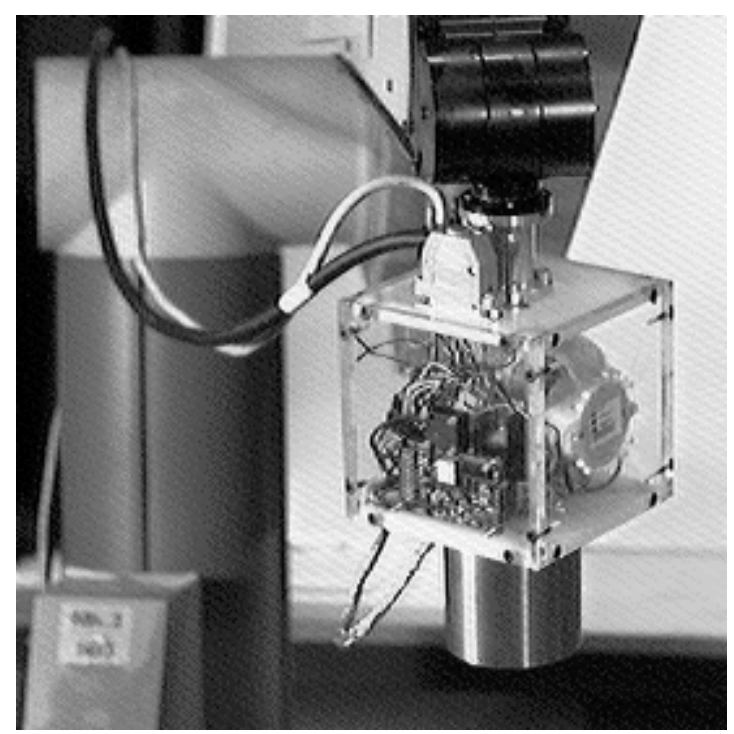

Fig.8. Experimental setup.

The Puma560 control was done using the real-time alter mode [12]. A pcboard performs low level communication protocol with 
the puma controller. A RS-232/RS-422 interface interconnects the two.

The acquired data can be viewed, stored and retrieved, and algorithms tested by adding modules to the INERworkbench written in the $*$.m matlab format.

\section{Conclusions}

The article presented an inertial system for land vehicles. The sensors, their mechanical mount and the processing pcboard were described. The characteristics and the functionality of each sensor were analyzed. Particular emphasis was given to the design of a magnetic shield for the fluxgate compass.

The algorithm, under testing, was designed for use in vehicles moving in a $2 \mathrm{D}$ plane. This kind of navigation is well adapted for mobile platforms.

Future improvements are planned for the system, such as the study of its errors and the improvement of the navigation algorithm using kalman filtering techniques.

\section{References}

[1] T. Viéville and O. D. Faugeras, "Computation of Inertial Information on a Robot", Fifth International Symposium on Robotics Research, Hirofumi Miura and Suguru Arimoto editor, MIT-Press, pages 57-65, 1989.

[2] B. Barshan, "Orientation Estimate for Mobile Robots using Gyroscopic Information", Proc. IROS'94, pages 18671874, 1994.

[3] K. Komoriya and E. Oyama , "Position Estimation of a Mobile Robot Using Optical Fiber Gyroscope (OFG)", Proc. IROS'94, pages 143-149, 1994.

[4] K. Rintanen, I. Kauppi, K. Koskinen and A. Koskinen, "Inertial Navigation for Mobile Robots by Redundant Low-cost Gyrometers".

[5] S. Cooper and H. Durrant-Whyte, "A Kalman Filter Model for GPS Navigation of Land Vehicles", Proc. IROS'94, pages 157-163, 1994.

[6] Y. Tonouchi, T. Tsubouchi and S. Arimoto, "Fusion of Dead-reckoned Positions with Workspace Model for a Mobile Robot by Bayesian Inference", Proc. IROS'94, pages 1347-1354, 1994.

[7] Murata MFG. Co, Ltd, "Piezoelectric Vibrating Gyroscope Gyrostar", Cat.No.S34E, 1991.

[8] T. J. Peters, "Automobile Navigation Using a Magnetic Flux-Gate Compass",IEEE Transactions on Vehicular Technology, pages 41-47, VOL. VT-35, No. 2, May 1986.

[9] KVH Industries, Inc, "C100 Compass Engine Technical Manual", KVH Part No. 54-0044, Revision G, May 1993.
[10] H. W. Ott, "Noise Reduction Techniques in Electronic Systems", John Wiley \& sons, 2nd edition, pages 159-201, 1988.

[11] D. R. J. White, "Electromagnetic Shielding Materials and Performance", Don White Consultants, 2nd edition, 1980.

[12] Unimation Incorporated, "Part 3 - Real-time Path Control, Programming Manual", User's Guide to VAL II 398T1, 1984. 